\title{
RELATIONSHIP OF JOINT BEHAVIOR WITH BODY COMPOSITION IN OLDER ADULT WOMEN AS DETERMINING FUNCTIONAL
}

\author{
Estrada - Bonilla, Yisel Carolina ${ }^{1}$; Ramírez- Villada, \\ Jhon Freddy ${ }^{2}$ \\ ${ }^{1}$ Grupo de Investigación en Cuerpo, Sujeto y \\ educación - Universidad Santo Tomás. \\ ${ }^{2}$ Grupo de investigación GICAEDS - Universidad \\ Santo Tomás
}

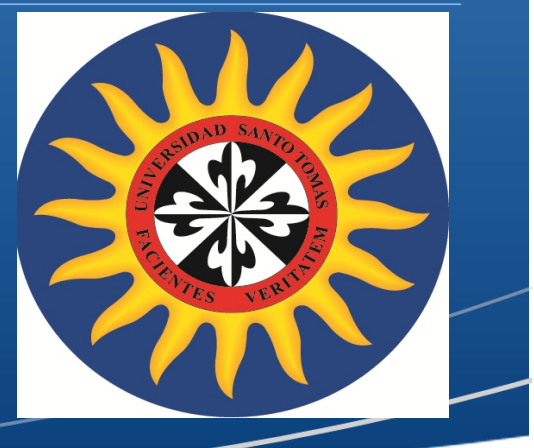

PURPOSE: The functionality in ederly is determined by components of body composition (fat mass/fat free mass), as well as issues related to physical fitness performance (muscle strength, joint mobility), exhibited by the elderly, making it independent for activities of the environment in which it operates. The objective of this research was to establish the relationship between joint behavioral characteristics (lower limb), with values of fat mass/fat free mass in older women doing the test jump " Squat Jump". The results were considered as indicators of the functionality in the population evaluated.

METHOD: Cross-sectional descriptive study type, where 29 women (mean age $58.93 \pm 3.01$ years), running jump " Squat Jump" is performed. Prior to the execution of the test, measurement of fat mass/fat free mass by bioimpedance (OMRON scale) is performed. Results of joint behavior in ankles, knees and hips in impulse jump phases 1 and 2, with the use of OptoGait tools (Microgate instruments) behavior are recorded. To determine the relationship between joint behavior (of lower limb) and body composition, Pearson coefficient is estimated by setting $r^{2}$ value for joint mobility range - JMR in ankle, knee and hip impulse phase 1 with fat mass/fat free mass, and JMR in ankle, knee and hip impulse phase 2 with fat mass/fat free mass.

RESULTS: Correlation between ankle JMR phase impulse 1 with fat mass $\left(r^{2}=-0.487 p=0.04\right)$. Correlation $r^{2}=0.475$ and $p=0.05$, between 1 phase impulse RMA ankles and fat - free mass. Correlation between impulse 1 RMA knee with fat mass $\left(r^{2}-0.247, p=0.07\right)$ phase. Correlation between impulse 1 phase RMA knee and fat - free mass $\left(r^{2}=0.193\right.$ and $\left.p=0.130\right)$. Correlation between ankle impulse 2 RMA and fat mass $\left(r^{2}=0.06\right.$ and $\left.p=-0417\right)$. Correlation between RMA ankle impulse 2 and fat - free mass $\left(r^{2}=0.567\right.$ and $\left.p=0.05\right)$.

CONCLUSIONS: Joint mobility range - JMR measured in impulse phases 1 and 2, not only depend on the willingness of the measure fat free mass, but also on the willingness of fat mass. The results show that a higher percentage of fat free mass, better performance and lower limb joint increased functionality of the individual most likely to adapt to the environment and functional independence.

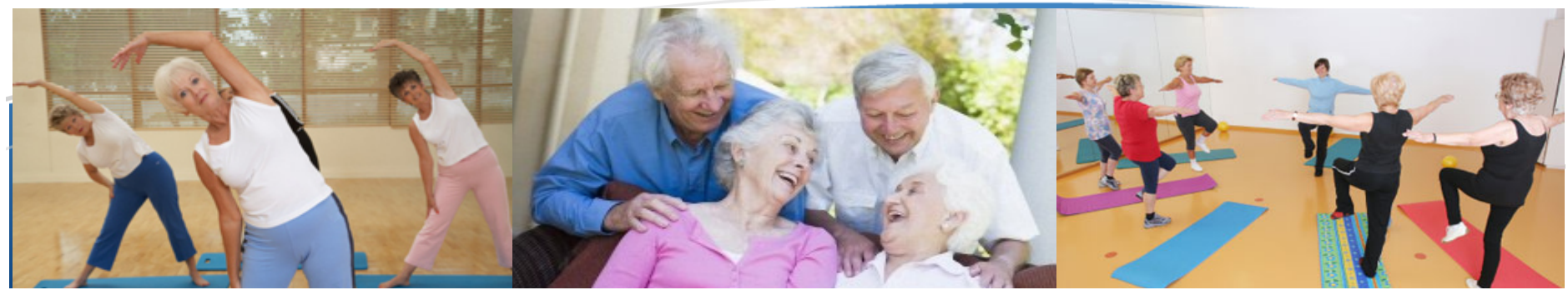




\section{Pellentesque habitant morbi tristique senectus et netus.}

Sed lacinia elit in neque mollis ullamcorper. Nulla facilisi. Ut risus erat, porta vitae, ultricies vel, dignissim vitae, erat. Suspendisse posuere felis id urna. Maecenas elementum tortor vel nibh. Praesent condimentum neque sit amet lacus. Sed nibh enim, viverra eget, faucibus quis, ornare sed, diam. In dui quam, blandit eget, consectetuer quis, facilisis a, quam. Vivamus sagittis consequat urna. Nunc mattis, pede id tempor consectetuer, mi justo rutrum risus, at euismod justo dolor sit amet dolor.

Integer ut nibh. In nec tellus quis massa consectetuer dapibus. Vestibulum sit amet orci ac quam viverra dictum. Vivamus sem. Curabitur aliquam, nisi in laoreet imperdiet, velit ligula vehicula urna, non congue neque mi a urna. Proin tellus. Sed blandit nulla ac turpis. Sed lacus. Etiam nibh lacus, porta et, dapibus vitae, dictum non, tellus. Phasellus fermentum, magna nec convallis tempus, sapien ipsum malesuada leo, eget interdum augue neque ac nibh.

Praesent sed augue. Pellentesque habitant morbi tristique senectus et netus et malesuada fames ac turpis egestas. Etiam semper lacus et risus. Suspendisse lorem elit, commodo non, sodales eget, rutrum gravida, metus. Mauris felis. Mauris porta, velit vel euismod tristique, odio nisi fermentum ante, non euismod elit massa tincidunt lectus.
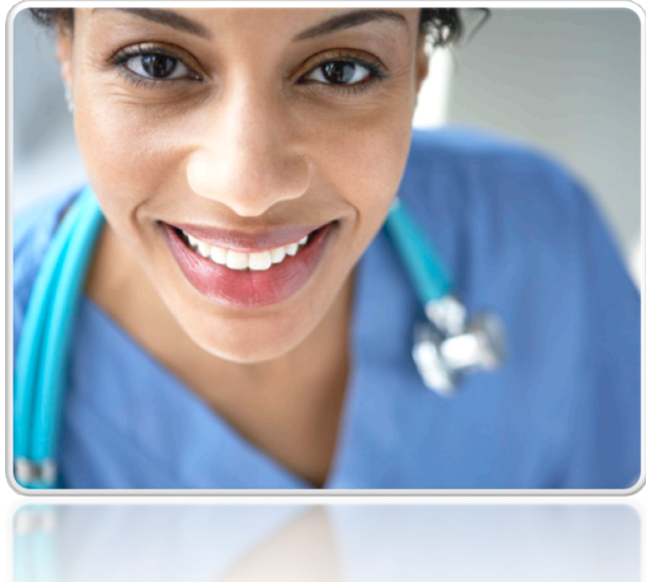

Nullam ac ipsum gravida sem placerat suscipit.

Aenean malesuada commodo sem. Praesent sit amet mi eget nulla suscipit condimentum. Curabitur ac libero. Class aptent taciti sociosqu ad litora torquent per conubia nostra, per inceptos hymenaeos. Integer rhoncus. Vestibulum vitae magna sed massa aliquet aliquam. Cras urna erat, fringilla sed, semper ut, sodales placerat, ante. Vivamus pulvinar mollis tellus. Nunc vitae pede a nisi sagittis pellentesque.

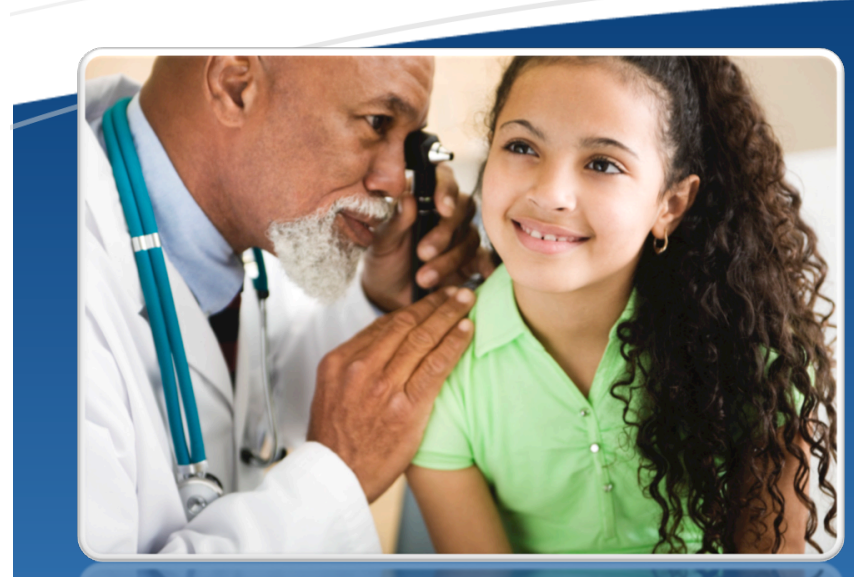

Nullam suscipit neque in ligula. Duis lectus. Curabitur ornare, magna iaculis sodales placerat, nisi tellus sollicitudin sapien, eu cursus pede justo ut nulla. Nullam in magna adipiscing ipsum fringilla iaculis.

\section{Aliquam scelerisque sagittis erat.}

Integer at diam. Ut vel quam vitae orci aliquam mattis. Nullam sem risus, dignissim non, tempus eget, laoreet et, est. Vestibulum eget tortor. Sed ante. In suscipit, metus sed luctus ornare, pede est dictum metus, sit amet posuere ante enim a ligula.

Nunc sodales. In ut enim. Quisque scelerisque pretium pede. Integer convallis. Praesent malesuada lacus sit amet lectus. Aliquam erat volutpat. Nullam lacus urna, faucibus et, placerat at, pulvinar id, massa. Morbi nec enim et leo placerat suscipit. Aenean sed massa non est vestibulum convallis. Mauris nec est sed est varius faucibus. 


\section{Praesent malesuada lacus sit amet lectus.}

Nam ac justo non erat ullamcorper vulputate. In hac habitasse platea dictumst. Curabitur eu lectus. Morbi sed quam ac tortor eleifend iaculis. Ut vel quam vitae orci aliquam mattis.

Morbi venenatis vestibulum magna. Pellentesque rutrum, dui at accumsan semper, massa ipsum nonummy dui, eget varius velit velit sed sapien. Suspendisse potenti.
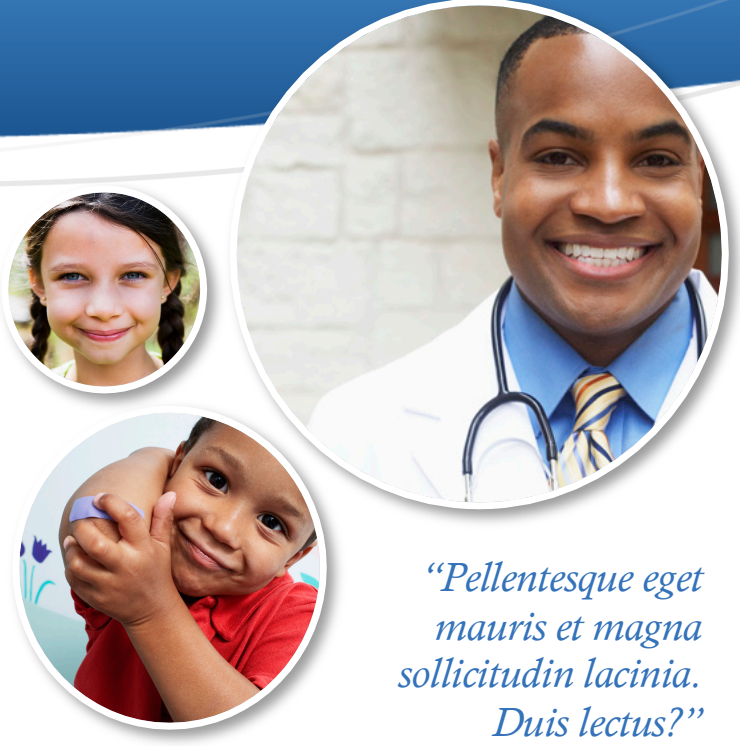

Etiam ornare mattis nulla. Mauris libero. Maecenas ultricies placerat sapien. Sed viverra tellus ut tortor. Lorem ipsum dolor sit amet, consectetuer adipiscing elit. Pellentesque nonummy, dui nec adipiscing pellentesque, neque purus volutpat felis, sit amet tristique tortor magna vitae augue.

Pellentesque habitant morbi tristique senectus et netus et malesuada fames ac turpis egestas.

Vestibulum eleifend, neque ut elementum volutpat, diam diam condimentum augue, id ullamcorper diam orci non odio. Sed eleifend nulla in urna. Aliquam erat volutpat. Cras dictum varius est.

Aenean malesuada odio lacinia mauris. Sed congue dui hendrerit orci. Proin luctus mauris in purus. Vivamus convallis pretium pede. Nullam at nunc id sem fringilla tristique. Integer iaculis neque quis magna. Phasellus semper. Vivamus vulputate.

\section{Fusce nunc diam, nonummy tristique, viverra} id, ultricies a, libero.

Aenean sed dui. Vestibulum tortor arcu, gravida sollicitudin, tempor sit amet, fermentum nec, eros. In nonummy diam id pede. Nunc at turpis. Duis bibendum. Integer ipsum diam, commodo in, rutrum eu, suscipit quis, magna. Sed vitae quam. Nulla facilisi.

Donec fermentum egestas arcu.

Cras adipiscing nulla sit amet lorem. Ut tellus augue, faucibus facilisis, scelerisque quis, dignissim ac, justo. Morbi non pede suscipit elit feugiat rhoncus. Ut sit amet libero. Donec felis mauris, pretium eget, congue nec, fermentum eu, erat. Donec metus purus, pharetra et, euismod sed, consectetuer quis, tellus. Duis laoreet consectetuer sapien. Proin a tellus sit amet elit porta iaculis. Suspendisse fringilla neque et est. Sed imperdiet dapibus mauris.

Curabitur venenatis magna vel urna. Donec sed eros nec ligula commodo porttitor. Sed viverra arcu nec diam. Vestibulum ut dolor sed quam porttitor euismod. Class aptent taciti sociosqu ad litora torquent per conubia nostra, per inceptos hymenaeos.

Pellentesque ultricies erat at purus. Duis vel velit. Curabitur fermentum dui eget urna. Curabitur accumsan odio at pede. Nam risus dui, venenatis ut, rhoncus vel, ullamcorper in, leo. Aenean lobortis blandit ante. Aliquam erat volutpat. Proin aliquam, lacus id rhoncus vehicula, enim arcu molestie tellus, a interdum nisi nunc ac eros. Donec magna. Aliquam erat volutpat. Cras sed lectus nonummy nibh convallis lobortis. Aliquam consequat arcu vel enim. Etiam placerat, pede non sodales egestas, diam mi tempor ipsum, a laoreet urna nunc vel augue. Vivamus mi. Cras at tellus in lectus fermentum lobortis. Donec ornare faucibus urna. Etiam ligula turpis, vestibulum sit amet, hendrerit a, ullamcorper at, ipsum. 
In hac habitasse platea dictumst. Donec justo. Aenean adipiscing lectus ac libero. Vivamus tristique. Nunc euismod. Proin dolor orci, volutpat a, iaculis mollis, elementum sed, sapien. Pellentesque quis elit. Aliquam sed arcu. Nunc tempor sollicitudin augue. Etiam enim. Aenean quis sem.

Mauris rutrum. Class aptent taciti sociosqu ad litora torquent per conubia nostra, per inceptos hymenaeos. Nullam dictum lobortis metus. Cras imperdiet interdum lorem. In congue fermentum turpis. Fusce vulputate lacinia enim. Suspendisse potenti. Praesent eleifend, ante quis bibendum mattis, quam felis eleifend nulla, et congue elit urna ac lorem. Suspendisse potenti. Morbi placerat lacus nec turpis. Aliquam turpis urna, euismod sed, ultricies ac, suscipit et, velit.

Nullam consectetuer, purus ut tempus euismod, ipsum ante volutpat purus, eget vulputate erat nibh vitae eros. Donec varius mauris vel enim. Duis nunc quam, vulputate nec, tincidunt sit amet, dignissim sit amet, elit. Donec congue nibh non odio. Curabitur ut elit vitae lorem egestas mollis. Mauris bibendum malesuada tortor. Curabitur adipiscing, lacus sit amet condimentum dapibus, pede nunc dictum enim, sit amet lobortis tortor odio vel nisi. Fusce eu velit a metus sollicitudin porta. Nam vel justo. Ut neque nisi, consequat id, pretium at, semper in, diam. Vestibulum aliquam mi id purus iaculis ullamcorper. Morbi ornare justo et magna. Aliquam ipsum orci, placerat nec, eleifend at, malesuada at, mi.

Sed porttitor cursus mi. In hac habitasse platea dictumst. Maecenas vel sem quis dui ultricies semper. Fusce lectus. Morbi fringilla augue eu nisl. Etiam bibendum. Fusce dictum adipiscing sapien.

Pellentesque habitant morbi tristique senectus et netus et malesuada fames ac turpis egestas. Donec pulvinar rhoncus dolor. Integer ac metus. Mauris id quam. Praesent iaculis, dolor eu sollicitudin fringilla, sapien nisl facilisis nisl, et ullamcorper urna magna quis orci. Etiam at libero. Integer pulvinar justo tincidunt metus. Proin vel felis sed neque laoreet posuere. Sed scelerisque egestas magna. Quisque eleifend erat quis nisi. Vestibulum imperdiet, urna at volutpat egestas, felis odio dapibus nisl, sed posuere nunc enim quis magna. Integer in magna. Suspendisse id justo.

Suspendisse vulputate lacus eget lectus. In ac massa. Donec sed diam. Cras egestas interdum libero. Vivamus tincidunt eros ac augue commodo ultrices. Curabitur luctus scelerisque leo.

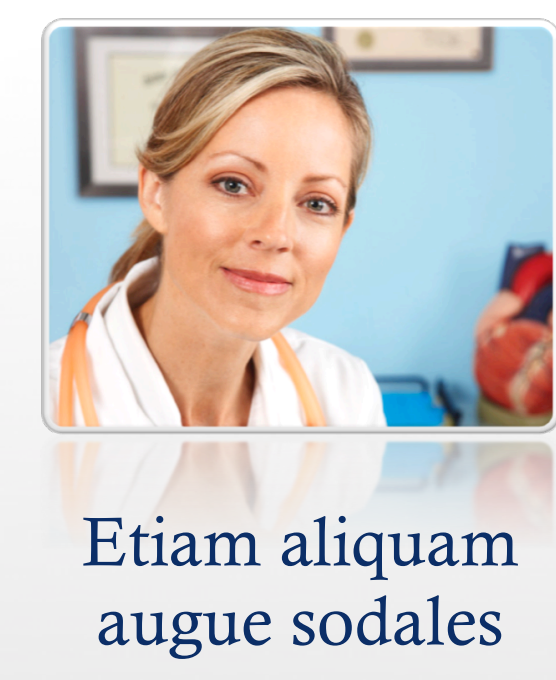

Integer sodales. Quisque id purus quis erat bibendum eleifend. Cras tincidunt ultrices ipsum. Suspendisse ut nulla. Praesent velit urna, eleifend et, varius eget, viverra varius, libero. Proin tincidunt viverra lectus. Sed rutrum, ante quis congue iaculis, turpis tortor posuere ligula, ut venenatis dolor purus at diam. Vivamus ac orci sit amet mi volutpat interdum. Etiam id odio. In a lorem. In condimentum metus vel augue. Vivamus semper imperdiet sem. Aliquam dolor ante, congue id, pretium sodales, lacinia eu, ligula. Ut non metus. Suspendisse laoreet. Integer adipiscing, justo a ultricies imperdiet, quam augue elementum augue, ac placerat mauris justo vitae nisi.

Praesent fermentum nulla vel elit. Donec aliquam dui quis sem ornare mattis. Vivamus sit amet odio et eros luctus nonummy. Donec diam orci, convallis ultrices, ultrices vitae, eleifend id, nisl. Class aptent taciti sociosqu ad litora torquent per conubia nostra, per inceptos hymenaeos. Vestibulum quis eros ac augue egestas accumsan. Nam libero libero, venenatis quis, sodales quis, dictum sagittis, dui. Fusce euismod tempor quam. Donec sit amet nunc ut diam consequat faucibus. In elit velit, facilisis eget, auctor vel, venenatis sed, sapien. Maecenas enim nunc, ultrices vitae, vulputate 
Quisque imperdiet purus quis orci. Ut placerat erat commodo libero. Praesent imperdiet. Sed bibendum velit nec ipsum. Sed quam metus, posuere ac, scelerisque at, vulputate sed, augue. Ut rhoncus vehicula leo. Quisque sem turpis, dignissim sit amet, ullamcorper vitae, condimentum id, metus. Nulla elit. Vestibulum mattis, purus et tempor tempor, purus elit rhoncus enim, eget mattis arcu odio ac odio. Mauris commodo. Maecenas eget eros ut felis accumsan ullamcorper. Nulla nec nunc. Aenean auctor, leo vel faucibus tincidunt, nulla dui ullamcorper mauris, eu porta felis metus non orci. Donec id tellus id orci porta pellentesque. Nam quis eros. Donec orci justo, viverra in, facilisis ut, euismod nec, libero.

\section{Donec sem nisi, tincidunt nec, congue sed,} suscipit vitae, justo.

Vivamus metus. Ut turpis augue, dictum condimentum, vestibulum quis, dictum luctus, velit. Aliquam cursus tortor quis lectus. Praesent semper nunc porttitor nibh. Sed pede. Vivamus nisi mi, luctus scelerisque, tristique a, mattis at, massa. Morbi vulputate risus ac leo. Praesent et sapien. Nulla eget felis. Morbi ipsum. Maecenas id ante eu tortor consectetuer feugiat. Duis libero. Donec malesuada ante vitae est. In tortor tortor, tincidunt eu, convallis sit amet, blandit vitae, mauris. Vestibulum sit amet ante. Aenean iaculis elementum enim. Fusce iaculis, elit vitae viverra venenatis, lectus elit viverra quam, vel scelerisque lectus augue eu est.

Cras accumsan leo a neque. Vestibulum egestas volutpat libero. Aliquam porta sodales justo. Suspendisse vel quam. Quisque ultrices fermentum odio. Nullam tempor vehicula enim. Nam justo elit, pellentesque quis, placerat sed, fermentum at, dui. Vestibulum nonummy laoreet metus. Donec consectetuer tortor in lorem. Duis mattis nisl ac ligula. Aenean sed orci id pede imperdiet consectetuer. Donec est. Nam commodo. Fusce quam sapien, ultricies quis, dictum id, commodo ut, erat. Sed posuere viverra arcu.

Vivamus vel sem eu massa sollicitudin congue. Aliquam tellus. Etiam neque lorem, congue vel, aliquet eget, auctor ac, dui. Nullam feugiat consectetuer enim. Ut vulputate erat eget lorem. Sed porta. Vivamus pharetra. Fusce tempor faucibus erat. Duis turpis. Fusce mollis fringilla nulla. Donec nisi magna, pretium vitae, interdum et, consectetuer sed, magna. Vivamus nec diam. Maecenas ac magna at sapien auctor consequat. Praesent iaculis mi vitae lorem.

In hac habitasse platea dictumst. Nulla odio. Aliquam ullamcorper. Praesent ipsum enim, rutrum et, pulvinar sed, tempor sed, metus. Pellentesque sodales. Quisque nunc. Aliquam commodo neque vel turpis. Donec et dolor et arcu semper pellentesque. Aliquam erat volutpat. Etiam eu ligula. Praesent quis quam. Nulla rutrum, magna sed gravida faucibus, purus magna bibendum purus, at bibendum ipsum mi eu risus.

Donec condimentum condimentum justo. Nullam sit amet turpis et eros tempor feugiat. Nullam laoreet lacus eget orci. In scelerisque nulla eget dui. Sed faucibus, lectus vitae pulvinar egestas, tortor velit porta nisi, id feugiat turpis mi sit amet felis. Vestibulum neque lorem, pretium ac, condimentum nec, malesuada et, massa. Donec nonummy bibendum diam.

Quisque sit amet odio. Pellentesque velit turpis, aliquam eu, ultricies vel, accumsan sit amet, quam. Praesent vulputate, arcu id convallis pellentesque, diam justo consequat massa, non rutrum odio ipsum ac magna. Quisque iaculis volutpat velit. Duis sed tortor sed turpis adipiscing euismod. Aliquam nec justo. Sed viverra ante eget sapien. Ut sodales adipiscing neque. Donec eu felis quis justo vehicula eleifend. Vivamus ultrices faucibus leo. Duis eu elit. Pellentesque lacus mauris, cursus vel, tempor ac, vestibulum et, lacus. Suspendisse ut 


\section{Lorem ipsum dolor sit amet, consectetuer adipiscing elit.}

Proin consequat. Donec et sem. Nullam in lorem. Vestibulum et est. In ullamcorper, quam nec dictum interdum, neque enim gravida tortor, id congue purus neque sit amet elit. Nunc gravida malesuada ante. Proin aliquet nisl ut libero. Sed quam. Lorem ipsum dolor sit amet, consectetuer adipiscing elit. In enim ante, condimentum ac, mollis eu, lobortis sed, sapien.

Fusce eleifend gravida est. Duis malesuada scelerisque purus. Donec sapien turpis, accumsan non, dapibus ut, congue ut, ligula. Integer placerat quam nec elit. Lorem ipsum dolor sit amet, consectetuer adipiscing elit. Morbi dictum pretium erat. Integer rhoncus velit sit amet pede. Fusce eu arcu id felis dignissim gravida. Maecenas scelerisque accumsan orci. Aliquam metus purus, ullamcorper sit amet, vehicula ac, venenatis et, orci. Donec venenatis enim suscipit sem.

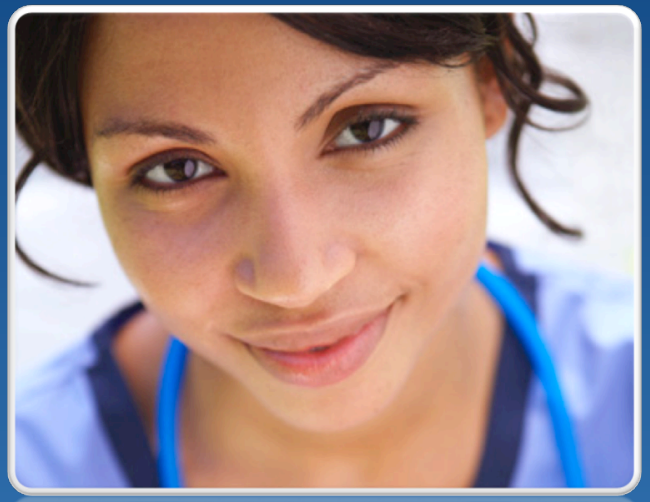

Vivamus id nisi vel purus gravida bibendum. Duis nec neque. In sem diam, convallis eleifend, rutrum id, rutrum et, justo. Cum sociis natoque penatibus et magnis dis parturient montes, nascetur ridiculus mus. Etiam malesuada eros at mi.

\section{Lorem Ipsum Dolor}

[Dirección]

[Ciudad], [Estado][Código postal]

\section{[Destinatario]}

Línea de dirección 1

Línea de dirección 2

Línea de dirección 3

Línea de dirección 4 